\title{
Ácidos Orgânicos e Compostos Clorados para Controle de Salmonella spp. em
} frangos

\section{Sandra Zabot ${ }^{1}$, Janice Ruschel ${ }^{2}$, Cristiane Michele Marchesi ${ }^{3}$, Alexandre da Trindade Alfaro ${ }^{4}$, Tereza Cristina Rocha Moreira de Oliveira ${ }^{5}$, Elisabete Hiromi Hashimoto ${ }^{6}$}

O objetivo deste trabalho foi avaliar a atividade de ácidos orgânicos e compostos clorados comerciais para o controle de Salmonella spp. A concentração inibitória mínima (CIM) do dióxido de cloro, dicloro isocianurato de sódio e ácidos tricloro isocianúrico, lático e peracético foi determinada para cepas padrões de $S$. Enteretidis, $S$. Typhimurium e a $S$. Heidelberg e avaliada com 54 cepas isoladas de frigorífico. O produto com melhor desempenho foi avaliado em água de chiller artificialmente contaminada com Salmonella spp. Entre os clorados, apenas o dicloro isocianurato $\left(60 \mathrm{mg} \cdot \mathrm{L}^{-1}\right)$ foi capaz de inibir Salmonella spp. A CIM dos ácidos lático e peracético variou de 0,5 a 2,0\%. Os ácidos peracético $(1,0 \%)$ e lático $(2,0 \%)$ foram capazes de inibir 98,14 e 100\% das cepas isoladas, respectivamente. O ácido lático a 2,0\% foi capaz de inibir completamente o crescimento das três cepas padrões de Salmonella spp. inoculadas na água do chiller. Os dados reforçam a necessidade de discussões para regulamentar o uso do ácido lático na tecnologia de abate de aves.

Palavras-chave: ácido lático, ácido peracético, dicloro isocianurato de sódio, Salmonella spp., CIM.

\section{Organic Acids and Chlorine Compounds for Control of Salmonella spp. in Poultry}

The aim of this work was to evaluate the activity of organic acids and commercial chlorinated compounds to control Salmonella spp. The minimum inhibitory concentration (MIC) of chlorine dioxide, sodium dichloro isocyanurate and trichloroisocyanuric, lactic and peracetic acids was determined using ATCC strains of $S$. Enteretidis, S. Typhimurium and S. Heidelberg and 54 strains isolated from the poultry slaughterhouse. Latic acid was evaluated in chiller water artificially contaminated with Salmonella spp. Among chlorinated agentes, only sodium dichloro isocyanurate (60 mg. $\mathrm{L}^{-1}$ ) was able to inhibit Salmonella spp. The MIC of the lactic and peracetic acids ranged from 0.5 to $2.0 \%$. Peracetic (1.0\%) and lactic acid $(2.0 \%)$ were able to inhibit 98.14 and $100 \%$ of the isolated strains, respectively. Lactic acid at $2.0 \%$ was able to completely inhibit the growth of the three ATCC strains of Salmonella spp. inoculated in the chiller water. The data reinforce the need for discussions to regulate the use of lactic acid in poultry slaughter technology.

Keywords: lactic acid, peracetic acid, dichloro isocyanurate, Salmonella spp., MIC.

\footnotetext{
1 - Mestre em Tecnologia de Alimentos. Programa de Pós-Graduação em Tecnologia de Alimentos na Universidade Tecnológica Federal do Paraná, Câmpus Francisco Beltrão.

2 - Tecnóloga de Alimentos. Universidade Tecnológica Federal do Paraná, Câmpus Francisco Beltrão

3- Doutora em Engenharia de Alimentos pela Universidade Regional Integrada do Alto Uruguai e das Missões Câmpus Erechim

4- Doutor em Ciência e Tecnologia Agroindustrial

5- Doutora em Ciência de Alimentos e professora do Programa de Pós-Graduação em Ciência de Alimentos da Universidade Estadual de Londrina.

6- Mestre e Doutora em Ciência de Alimentos e professora do Programa de Pós Graduação em Tecnologia de Alimentos da Universidade Tecnológica Federal do Paraná.

Endereço para correspondência: Universidade Tecnológica Federal do Paraná, Câmpus Francisco Beltrão; Programa de Pós Graduação em Tecnologia de Alimentos, Depto

Acadêmico de Química e Biologia, Caixa Postal 135, CEP 85601970, Francisco Beltrão -PR.
} 


\section{INTRODUÇÃO}

No Brasil, o cenário do mercado da carne de frango modernizou-se em decorrência, principalmente das exportações. O mercado externodemandou melhorias no sistema para o controle microbiológico, sobretudo quanto à contaminação por Salmonella spp ${ }^{[1]}$. Salmonella enterica sorovar Typhimurium, $S$. Enteritidis e $S$. Heidelberg são consideradas sorovares encontradas no trato intestinal de diferentes espécies de animais ${ }^{[2]}$.

A carne de aves e seus derivados estão entre os principais alimentos envolvidos em surtos de infecções alimentares por Salmonella spp. Estes surtos ocorrem em decorrência do preparo inadequado e da contaminação cruzada em cozinhas domiciliares e industriais. A contaminação das carcaças de frango pode acontecer pela presença do microrganismo no ambiente de criação das aves, ou ainda, pela disseminação nas carcaças durante as operações de abate ${ }^{[3]}$.

De acordo com Doyle \& Erickson ${ }^{[4]}$, para reduzir a contaminação por patógenos de origem alimentar, pode ser adotada uma combinação de estratégias de intervenção. Neste sentido, Buncic \& Sofos ${ }^{[5]}$ relatam tratamentos que podem ser aplicados em carcaças de aves ou em suas partes, tais como água, vapor e soluções químicas (ácido lático ou acético, compostos clorados dentre outros). Tratamentos com ácidos orgânicos são de baixo custo, simples e rápidos e têm mostrado eficiência em muitos casos. Além disso, por serem considerados seguros, vários ácidos orgânicos não têm estabelecido o limite máximo de ingestão diária. Estas características tornam interessante o uso destes agentes em produtos cárneos. No entanto, a possibilidade de mudanças sensoriais como cor e sabor deve ser consideradana aplicação do composto ${ }^{[6]}$.

O uso de ácidos orgânicos como descontaminantes de carcaças não é permitido pela legislação brasileira. No entanto, nos Estados Unidos, por exemplo, os ácidos orgânicos podem ser utilizados desde que sejam aprovados pelas autoridades sanitárias competentes, e utilizados em concentrações limitadas ${ }^{[7]}$.

A produção avícola brasileira esta em constante crescimento e o setortem investidoem pesquisa e desenvolvimento para produção e fornecimento de alimentos seguros e de elevada qualidade. Para que isso seja possível, se faz necessária a avaliação da eficácia de novas técnicas que auxiliem e promovam o controle microbiológico. Assim, este trabalho objetivou avaliar a eficácia de compostos clorados e ácidos orgânicos no controle da contaminação de carne de frango por Salmonella spp.

\section{MATERIAL E MÉTODOS}

Salmonella enterica sorovar Heidelberg (ATCC 8326), S. Typhimurium (ATCC 14028) e S. Enteritidis (ATCC 13076) foram utilizadas comocepas padrões. Foram testadas também 54 cepas de Salmonella spp. isoladas de um frigorífico no período de junho a setembro de 2014, que opera com Serviço de Inspeção Federal e sistema APPCC implantado, conforme requisitos do Ministério da Agricultura, Pecuária e Abastecimento ${ }^{[8]}$ e controle de Salmonella spp. em carcaças de frangos ${ }^{[9]}$. A realização das quatro etapas sucessivas necessárias para a detecção de Salmonella spp. (pré-enriquecimento, enriquecimento seletivo, plaqueamento e confirmação bioquímica e sorológica) seguiram a metodologia de referência ISO 6579:2002 ${ }^{[10]}$ Microbiology of Food and Animal Feeding Stuffs - Horizontal Method for detection of Salmonella spp.

Para as análises de atividade antimicrobiana foram testados os compostos clorados comerciais dicloro isocianurato de 
sódio $\left(60 \%, \mathrm{C}_{3} \mathrm{Cl}_{2} \mathrm{~N}_{3} \mathrm{O}_{3} \mathrm{Na}\right.$ comercial) e ácido tricloro isocianúrico $\quad\left(90 \%, \quad \mathrm{C}_{3} \mathrm{Cl}_{3} \mathrm{~N}_{3} \mathrm{O}_{3}\right.$ comercial). As concentrações máximas testadas dos compostos clorados basearam-se nas recomendações dos fornecedores de cada composto, sendo: dicloro isocianurato de sódio $60 \mathrm{mg} . \mathrm{L}^{-1} \mathrm{e}$ ácido tricloro isocianúrico $30 \mathrm{mg} . \mathrm{L}^{-1}$. Os ácidos orgânicos testados consistiram de ácido lático p.a. (85\%, $\mathrm{C}_{3} \mathrm{H}_{6} \mathrm{O}_{3}$, p.a.), e ácido peracético comercial $\left(15 \%, \mathrm{C}_{2} \mathrm{H}_{4} \mathrm{O}_{3}\right.$ comercial) em concentrações que variaram de 0,1 a 3,0\%.

Um volume de $100 \mu \mathrm{L}$ de cada cultura de Salmonella ATCC padronizada a $10^{8}$ UFC.mL ${ }^{-1[1]]}$ foi adicionada em 9,9 mL de cada solução teste de antimicrobiano (solução aquosa adicionada de leite, 1:10). Assim, considerando a diluição (1:100) os tubos cotendo $10^{6}$ UFC. $\mathrm{mL}^{-1}$ de cada cepa foram homogeneizados e mantidos a $25{ }^{\circ} \mathrm{C}$. Após o tempo de exposição de 10, 15, e $20 \mathrm{~min}$, uma alíquota de $10 \mu \mathrm{L}$ de cada teste foi inoculadaem tubo contendo $5 \mathrm{~mL}$ de caldo BHI (Himedia, Índia), em triplicata. Em seguida os tubos foram incubados a $35{ }^{\circ} \mathrm{C}$ por $72 \mathrm{~h}{ }^{[12]}$. Foi considerada a concentração inibitória mínima (CIM) a menor concentração que não apresentou turbidez, sendo esta confirmada pelasemeadura de $100 \mu \mathrm{L}$ em meio seletivo para Salmonella spp. (ágar SS, Himedia, Índia).

Para avaliação da inibição das 54 cepas selvagens isoladas do frigorífico, testou-se os compostos na CIM e no melhor tempo de exposição, a partir dos resultados dos testes com as cepas ATCC. As soluções ácidas foram preparadas com e sem leite. Para a análise foram transferidos $180 \mu \mathrm{L}$ de cada ácido na CIM em cada poço da microplacade polestireno, em seguida adicionou-se $20 \mu \mathrm{L}$ das cepas padronizadas $10^{7} \mathrm{UFC} \cdot \mathrm{mL}^{-1}$ nos poços contendo os compostos em triplicata, e expostas por $20 \mathrm{~min}$. Em seguida foram transferidos $20 \mu \mathrm{L}$ de cada teste em outra microplaca contendo $180 \mu \mathrm{L}$ de caldo BHI e incubado a $37^{\circ} \mathrm{C}$, resultando em um inóculo de $10^{6}$ UFC.mL $\mathrm{m}^{-1}$. Após o período de incubação foram realizadas as leituras da absorbância a
$620 \mathrm{~nm}$ em fotômetro (Thermoplate TP Reader). Nos poços em que não houve turbidez, ou seja, onde houve a atividade antimicrobiana frente à Salmonella spp., foram inoculados $100 \mu \mathrm{L}$ para meio seletivo para Salmonella para a confirmação da inibição do microrganismo.

O pH foi medido com auxílio de pHmetro (Mettler Toledo, Estados Unidos) em $10 \mathrm{~mL}$ de cada solução teste cotendo cada agente: solução aquosa sem adição de leite e com a adição de leite $(1 \mathrm{~mL}$ leite UHT integral $+9 \mathrm{~mL}$ da solução aquosa). A constante de dissociação e molaridade de cada ácido orgânico, foi utilizada para o cálculo do $\mathrm{pH}$ teórico.

Um total de dois litros de água de chiller foi obtida de um abatedouro de frangos cujo o sistema de resfriamento possui cloração de 0,3 a 0,5 mg. $\mathrm{L}^{-1}$. A amostra de água foi coletada do último estágio do sistema de resfriamento em virtude deste estágio conter a menor quantidade de matéria orgânica e reter as carcaças por um tempo mais prolongado em relação aos demais estágios (cerca de $40 \mathrm{~min}$ ). A água foi coletada em frascos estéreis sob condições assépticas. Imediatamente após a coleta, os frascos de água foram transportados e encaminhados em recipiente isotérmico para o laboratório. As cepas padrões de Salmonella ATCC 14028, 13076 e 8326 foram padronizadas na concentração de $10^{9}$ UFC. $\mathrm{mL}^{-1}(625 \mathrm{~nm})^{[11]}$. Uma alíquota de $100 \mu \mathrm{L}$ de cada cepa foi diluída em $100 \mathrm{~mL}$ de água de chillera dicionada de ácido lático com concentração final de 2,0\%. A concentração do inóculo após diluição foi de $10^{6}$ UFC. $\mathrm{mL}^{-1}$. O controle consistiu de água de chiller sem adição de ácido lático. As amostras foram homogeneizadas e mantidas à temperatura de $4^{\circ} \mathrm{C}$ por $40 \mathrm{~min}$.

As alíquotas de água foram homogeneizadas e manteve-se o contato da água com o composto e a cepa padrão durante 40 min em temperatura de $4{ }^{\circ} \mathrm{C}$, simulando as condições reais da etapa de resfriamento do 
frigorífico. Para a contagem de Salmonella spp. foram realizadas diluições seriadas até $10^{-5}$. Um volume de $100 \mu \mathrm{L}$ de cada diluição foi inoculada em superfície de placas de Petri contendo ágar BPLS (ágarverde brilhante, vermelho de fenol, lactose, sacarose, Merck, Alemanha). As placas foram então incubadas à $36 \pm 1{ }^{\circ} \mathrm{C}$ por $48 \mathrm{~h}$. Posteriormente, procedeuse com a contagem das colônias, as quais foram caracterizadas pela coloração rosa/vermelho claro, opacas, rodeadas por zonas vermelhas brilhantes. Os resultados foram expressos em $\mathrm{UFC} / \mathrm{g}^{[13]}$. Os ensaios foram realizados em triplicata.

\section{RESULTADOS E DISCUSSÃO}

O ácido tricloro isocianúrico (TCCA) comercial não apresentou atividade inibitória contra nenhuma das cepas de Salmonella ATCC testadas, mesmo na maior concentração testada (30 mg. $\left.\mathrm{L}^{-1}\right)$. Sabe-se que a aplicação do TCCA é mais comum para desinfecção de piscina e tratamento de água ${ }^{[14,15]}$. Gonçalves et al. ${ }^{[16]}$ relataram a aplicação de soluções aquosas em concentrações de 45 a 100 mg.L. $\mathrm{L}^{-1}$ em pH 3,5 por 15 min de exposição foi capaz de reduzir Listeria monocytogenes em 0,07 a 4,41 log de UFC em peitos de frango artificialmente contaminadas, com redução de 2,55 log a partir de $60 \mathrm{mg} . \mathrm{L}^{-1}$. Nesse estudo, oproduto contendo TCCA apresentoulenta dissolução, sendo este um dos fatores que pode ter prejudicado a eficiência do composto comercial, visto que o tempo máximo de exposição do ácido com as cepas foi de $20 \mathrm{~min}$. Aliado a este fato, destaca-se também que por apresentar uma estrutura química estável, o composto pode ter reagido lentamente com a matéria orgânica, representada pela adição do leite, o que levou a uma liberação mais lenta do ácido hipocloroso.

A presença de matéria orgânica afetou os valores de $\mathrm{pH}$ das soluções testadas. $\mathrm{O}$ TCCA nas concentrações de 2,5 a 30 mg. $\mathrm{L}^{-1}$ apresentou valores de $\mathrm{pH}$ menores para a solução aquosa (pH 4,55 a 3,01) que as soluções adicionadas de leite 1:10 (pH 6,66 a 5,77). A matéria orgânica, neste caso representada pela adição de leite resultou em valores de $\mathrm{pH}$ mais elevados.

Segundo Mokgatlaet al. ${ }^{[17]}$, a menor eficiência dos compostos clorados tem sido relacionada com o uso frequente dos mesmos, pois o uso prolongado pode resultar em tolerância aos compostos pelos microrganismos.

A Tabela 1 apresenta as concentrações inibitórias mínimas (CIM) dos ácidos lático (AL) e peracético (AP) e dicloro isocianúrico de sódio ( $\mathrm{NaDCC}$ ) para as cepas padrões de Salmonella spp. expostas às soluções ácidas por 10 a $20 \mathrm{~min}$ a $25^{\circ} \mathrm{C}$.

Tabela 1 - Concentração inibitória mínima de dicloro isocianurato, ácidos lático e peracético para controle de Salmonella spp.

\begin{tabular}{lcccc}
\hline $\begin{array}{l}\text { Salmonella spp. } \\
\text { (ATCC) }\end{array}$ & $\begin{array}{c}\text { Temp } \\
\text { o exp. } \\
\text { (min) }\end{array}$ & \multicolumn{3}{c}{$\begin{array}{c}\text { Concentração } \\
\text { inibitória mínima }\end{array}$} \\
\cline { 3 - 6 } & & $\begin{array}{c}\text { NaDCC } \\
\left(\mathrm{mg}^{-1} \mathrm{~L}^{-1}\right)\end{array}$ & $\begin{array}{c}\mathrm{AL} \\
(\%)\end{array}$ & $\begin{array}{c}\mathrm{AP} \\
(\%)\end{array}$ \\
\hline S.Typhimurium & 10 & $\mathrm{R}$ & 2,0 & 1,0 \\
$(14028)$ & 15 & $\mathrm{R}$ & 1,0 & 1,0 \\
& 20 & $\mathrm{R}$ & 1,0 & 1,0 \\
\hline S.Heidelberg & 10 & $\mathrm{R}$ & 2,0 & 1,0 \\
(8326) & 15 & 60 & 2,0 & 1,0 \\
& 20 & 60 & 2,0 & 1,0 \\
\hline S. Enteritidis & 10 & $\mathrm{R}$ & 0,5 & $\mathrm{R}$ \\
(13076) & & & & \\
& 15 & $\mathrm{R}$ & 0,5 & $\mathrm{R}$ \\
& 20 & 60 & 0,5 & 1,0 \\
\hline
\end{tabular}

NaDCC: Dicloro Isocianurato de sódio, AL: ácido lático e AP: ácido peracético. R: resistente.

A solução de $\mathrm{NaDCC}$ apresentou inibição de crescimento dos sorovares de Salmonella (ATCC 8326 e 13076), com exceção da $S$. Typhimurium, a qual revelou resistência ao composto testado em todas as concentrações e tempos avaliados. Salmonella Heidelberg e $S$. 
Enteritidis foram inibidos na concentração de 60 mg. $\mathrm{L}^{-1}$ durante os tempos de exposição de 15 e 20 min, respectivamente (Tabela 1 ).

É importante destacar que o $\mathrm{NaDCC}$ utilizado nos experimentos foi obtido comercialmente e as concentrações das soluções foram preparadas a partir do teor de cloro especificado pelo fornecedor. Ainda, o NaDCC em solução aquosa libera ácido hipocloroso e isocianurato de sódio, estes com ação antimicrobiana. O NaDCC apresenta uma estrutura química estável e que pode reagir lentamente com a matéria orgânica ${ }^{[16]}$, embora a diferença de $\mathrm{pH}$ entre as soluções aquosa $(\mathrm{pH}=6,95$ a 6,39) e adicionada de leite $(\mathrm{pH}=8,19$ $7,36)$ tenha sido mínima nas concentrações testadas (2,5 a 60 mg. $\left.\mathrm{L}^{-1}\right)$. No experimento a matéria orgânica representada pela adição do leite, pode ter levado a uma liberação mais lenta do ácido hipocloroso, sendo o NaDCC efetivo somente na maior concentração testada (60 mg.L' $\left.{ }^{1}\right)$.

O uso de $150 \mathrm{mg} \cdot \mathrm{L}^{-1} \mathrm{NaDCC}$ com tempo de exposição de $10 \mathrm{~min}$ foi relatado e reduziu cerca de 2,5 log UFC.mL ${ }^{-1}$ de uma suspensãode sorovares de Salmonella (S. Montevideo, $S$. Newport, S. Saintpaul e $S$. Typhimurium ATCC14028) a $10^{4}-10^{5}$ UFC por amostra de Jimaca um tipo de nabo mexicano ${ }^{[18]}$. Assim, a concentração máxima testada foi recomendada pelo fornecedor do produto comercial, no entanto é possível que o produto testado pudesse ter atividade também contra $S$. Typhimurium se aplicado em concentrações maiores.

O AP teve a atividade inibitória somente na concentração de $1,0 \%(\mathrm{pH} 2,79)$ a partir de 10 min de exposição para $S$. Typhimurium e $S$. Heidelberg, sendo que $S$. Enteritidis necessitou de maior tempo de exposição para ser inibida (20 min). A avaliação de 20 cepas de $S$. Heidelberg isolada de um frigorífico nos anos de 2005 e 2009 mostrou sensibilidade ao ácido peracético 1\% a partir de $5 \mathrm{~min}$ de exposição ${ }^{[3]}$. No presente trabalho o menor tempo de exposição testado frente à cepa ATCC 8326 foi de 10 min.
A avaliação de um produto comercial composto de ácido peracético $2 \%$, peróxido de hidrogênio $6 \%$ e ácido acético $22 \%$ apresentou maior atividade na ausência de matéria orgânica (soro bovino fetal). Embora este produto comercial diluído 1:200 tenha reduzidoem 8,64 log a contagem de $S$. Enteritidis em presença de matéria orgânica, em solução sem a adição de soro bovino fetal o microrganismo foi totalmente inibido ${ }^{[19]}$. No presente estudo, todas as concentrações testadas apresentaram valores de $\mathrm{pH}(\mathrm{pH} \leq 4,10)$ abaixo do valor do $\mathrm{pKa}$ do AP $(\mathrm{pKa}=8,20)$. No entanto, somente em valores de $\mathrm{pH}$ menores ou iguais a 2,79 o AP foi capaz de inibir o crescimento de Salmonella, indicando a interferência da matéria orgânica na ação antibacteriana do AP.

Uma menor contagem de $S$. Typhimurium foi observada em carcaças de frango tratada com 0,01\% (1 mg.mL $\left.\mathrm{mL}^{-1}\right)$ de AP quando comparado com cloro a $0,003 \%$ em água de chiller. No entanto, após 15 dias de estocagem, as carcaças tratadas com AP 0,01\% foram afetadas sensorialmente, resultando em carne com cor e odor desagradáveis ${ }^{[20]}$.

Machado et al. ${ }^{[21]}$, ao estudarem a susceptibilidade do AP em relação a Salmonella Typhimurium, S. Enteritidis e $S$. Bredeney, evidenciaram que na concentração de uso indicada pelo fabricante $(1 \%)$ e na metade dessa concentração $(0,5 \%)$, todos os microrganismos foram sensíveis nos tempos avaliados $(5,10,15 \mathrm{e}$ $20 \mathrm{~min})$. E ainda, verificaram que as concentrações de 0,3, 0,2 e 0,1\% indicaram maior resistência dos microrganismos $S$. Typhimurium e S. Enteritidis.

Entre as cepas padrões testadas, a Salmonella Enteritidis foi a mais sensível ao ácido lático (AL), sendo inibida à $0,5 \%(\mathrm{pH} 2,91) \mathrm{em}$ tempo exposição mínimo de 10 min., seguida da $S$. Typhimurium, a qual foi inibida após o tempo mínimo de $15 \mathrm{~min}$ quando exposta à $1,0 \%(\mathrm{pH}$ 2,57). S. Heidelberg foi a mais resistente ao AL requerendo 2,0\% (pH 2,33) nos tempos de 10 a 20 min. Embora não seja permitido pela 
legislação brasileira, o Departamento de Agricultura dos EUA ${ }^{[7]}$ Serviço de Segurança e Inspeção de Alimentos (FSIS) aprovou o uso de alguns ácidos orgânicos como tratamentos antimicrobianos para carcaças de carne bovina. $\mathrm{O}$ ácido láctico tem baixa toxicidade para os seres humanos e está listado como "geralmente reconhecido como seguro" - GRAS [22].

Em relação ao pH, constatou-se que para o ácido lático, as soluções testes nas concentrações inibitórias mínimas apresentaram valores de $\mathrm{pH}$ de $2,91(0,5 \%), 2,57(1,0 \%)$ e 2,33 $(2,0 \%)$, revelando-se inferiores ao $\mathrm{pKa}$ do $\mathrm{AL}$ $(\mathrm{pKa}=3,85)$. Enfatiza-se que o efeito bactericida dos ácidos orgânicos é ocasionado pela forma não dissociada, sendo esta mais predominante em valores de $\mathrm{pH}$ inferiores ao $\mathrm{pKa}^{[23]}$. O controle do $\mathrm{pH}$ é fundamental para a ação do ácido lático, em $\mathrm{pH}$ mais próximo a neutralidade $(\mathrm{pH} 5,0$ a $6,0)$ espécies de Salmonella podem apresentar mecanismo de adaptação ${ }^{[17]}$. A adição de leite no experimento revelou influência da matéria orgânica nos valores de $\mathrm{pH}$ do ácido lático. Os valores de $\mathrm{pH}$ foram inferiores nas soluções aquosas $\mathrm{pH}=2,62,2,31$ e 2,14 para as concentrações de $0,5, \quad 1,0$ e 2,0\%, respectivamente.

Os ácidos láticos e peracético nas concentrações testadas frente às 54 cepas de Salmonella spp. isoladas de frigorífico apresentaram resultados satisfatórios nas concentrações testadas (Tabela 2). AL na concentração de 2,0\% inibiu o crescimento de todas as cepas testadas, independente do tempo de exposição. O AP a 1\%, após 20 min., inibiu 98,14\% das cepas de Salmonella spp. isoladas de frigoríficos. O NaDCC por sua vez foi capaz de inibir menos da metade $(46,29 \%)$ das cepas.
Tabela 2 - Atividade antimicrobiana dosácidoslático eperacético, e dicloro isocianurato de sódio sobre a inibição de Salmonella spp. isoladas de frigorífico.

\begin{tabular}{|c|c|c|c|c|}
\hline \multirow{2}{*}{$\begin{array}{c}\text { Períod } \\
\text { o } \\
\text { (mês) }\end{array}$} & \multirow{2}{*}{$\begin{array}{c}\mathrm{N}^{\mathrm{o}} \\
\mathrm{de} \\
\text { cepas }\end{array}$} & \multicolumn{3}{|c|}{$\mathrm{N}^{\mathrm{o}}$ de Cepas sensíveis } \\
\hline & & $\begin{array}{l}\text { AL } \\
(2 \%)\end{array}$ & $\begin{array}{c}\text { AP } \\
(1,0 \%)\end{array}$ & $\begin{array}{c}\mathrm{NaDCC} \\
(60 \\
\left.\mathrm{mg} \cdot \mathrm{L}^{-1}\right)\end{array}$ \\
\hline Jun & 14 & 14 & 14 & 8 \\
\hline Jul & 13 & 13 & 12 & 7 \\
\hline Ago & 21 & 21 & 21 & 7 \\
\hline Set & 6 & 6 & 6 & 3 \\
\hline $\begin{array}{c}\text { Total } \\
(\%)\end{array}$ & 54 & $\begin{array}{c}54 \\
(100 \\
\%) \\
\end{array}$ & $\begin{array}{c}53 \\
(98,14 \%)\end{array}$ & $\begin{array}{c}25 \\
(46,29 \%)\end{array}$ \\
\hline
\end{tabular}

AL: ácido lático e AP: ácido peracético. $\mathrm{N}=54$

A Tabela 3 apresenta os resultados da aplicação de ácido lático em água de chiller artificialmente contaminada com Salmonella spp.O AL foi testado em água artificialmente contaminada com as cepas padrões de $S$. Heidelberg, $S$. Enteritidis e $S$. Typhimurium a 4 ${ }^{\circ} \mathrm{C}$, afim de se simular a etapa de chiller. Nestas condições o AL a 2,0\%, após $40 \mathrm{~min}$ de exposição, foi capaz de controlar completamente o desenvolvimento das cepas de Salmonella spp. testadas. Em comparação, o controle constituído somente por água resfriada de chillerna quala contagem das soravares se manteve entre 6,82 a $6,69 \log$ UFC. $\mathrm{mL}^{-1}$ (Tabela 3). Os resultados reforçaram a capacidade destes compostos de reduzir contaminações microbiológicas em sistemas de resfriamento. Mesmo diante da matéria orgânica presente na água de resfriamento, o ácido lático não perdeu sua efetividade, revelando também sua estabilidade. 
Tabela 3 - Aplicação de ácido lático em água artificialmente contaminada com Salmonella spp. (6 logde UFC.mL $\left.{ }^{-1}\right)$ a $4^{\circ} \mathrm{C}$.

\begin{tabular}{|c|c|c|}
\hline \multirow[t]{2}{*}{$\begin{array}{l}\text { Salmonella spp. } \\
\text { (ATCC) }\end{array}$} & \multicolumn{2}{|c|}{$\begin{array}{c}\text { Contagem de } \\
\text { Salmonellaspp. (log } \\
\left.\text { UFC.mL }{ }^{-1}\right)\end{array}$} \\
\hline & $\begin{array}{c}\mathrm{AL} \\
(2,0 \%)\end{array}$ & $\begin{array}{c}\text { Controle } \\
\text { (água de } \\
\text { Chiller) }\end{array}$ \\
\hline S. Heidelberg (8326) & N.D. & $6,69 \pm 0,05$ \\
\hline $\begin{array}{l}\text { S. Typhimurium } \\
(14028)\end{array}$ & N.D. & $6,77 \pm 0,03$ \\
\hline S. Enteritidis (13076) & N.D. & $6,82 \pm 0,04$ \\
\hline
\end{tabular}

UFC/mL: Unidade formadora de colônia por mililitro. ND - Crescimento Não Detectado

Embora o teste com água de chiller tenha sido realizado a $4{ }^{\circ} \mathrm{C}$ e somente na concentração de 2,0\% de AL, dependendo da sorovar contaminante é possível que concentrações menores fossem suficientes. Os testes para determinação da CIM in vitro foram conduzidos a $25{ }^{\circ} \mathrm{C}$ com respostas diferentes para as trêscepas testadas (Tabela 1), com CIM de $0,5,1,0$ e 2,0\%. Experimentos com desinfetantes comerciais compostos de ácido peracético e mistura de ácidos orgânicos testados frente a Salmonella spp em temperaturas de 10 e $30^{\circ} \mathrm{C}$ não apresentaram diferenças na ação antibacteriana destes compostos $^{[18]}$. A concentração testada de AL a $2,0 \%$ considerou a necessidade de garantir a ação antibacteriana independente do sorovar.

Além das diferentes respostas entre as sorovares, há de considerar a forma de aplicação do ácido lático. Em meio de cultura sólida o ácido lático a $0,1 \%$ foi capaz de inibir completamente o desenvolvimento de Salmonella Enteritidis, $S$. Anatum e $S$. Typhimurium $\left(10^{8} \mathrm{UFC}\right)^{[24]}$. Já a pulverização de ácido lático $(0,5,1,1,5$ e $2 \%)$ ocasionou reduções de 0,4 a $1,4 \log$ UFC.cm ${ }^{-2}$ de $S$. Typhimurium na superfície de lombos suínos ${ }^{[25]}$. Além disso, há de ressaltar a capacidade de Salmonella spp. formar biofilmes e afetar a ação do agente antimicrobiano ${ }^{[26]}$. Os experimentos conduzidos neste trabalho foram realizados em soluções aquosas, com inóculo de células de Salmonellaspp., nos tempos testados não observou-se a formação de biofilmes. Neste sentido, deve-se consideraçõesesta possibilidade, sendo necessários ajustes nas concentrações a serem aplicadasdependendo do uso pretendido destes agentes.

\section{CONCLUSÃO}

Entre os compostos clorados comerciais, somente o dicloro isocianurato foi capaz de inibir Salmonella spp. Considerando a possibilidade de tolerância microbiana ao cloro utilizado no controle de patógenos, a disposição de alternativas de controle que sejam seguras, eficazes e biodegradáveis se faz necessária. Os ácidos peracético e lático mostraram-se eficazes inibindo grande parte das cepas de Salmonella spp. testadas. O ácido lático (GRAS) teve seu efeito confirmado quando aplicados em água de chiller artificialmente contaminada com Salmonella spp. Embora não seja permitido pela legislação brasileira, o uso do ácido lático em abatedouro de frangos é prática adotada nos Estados Unidos da América. Diante disso, discussões são necessárias para regulamentar o uso deste ácido em suporte a programas de biosseguridade na linha de abate de aves.

\section{REFERÊNCIAS}

[1] Von Rückert, DAS, Pinto, PSA., Santos, BM, Moreira, MAS, Rodrigues, ACA. Pontos críticos de controle de Salmonella spp. no abate de frangos. Arquivo Brasileiro de Medicina Veterinária e Zootecnia. 2009; 61 (2):326-330.

[2] Guibourdenche M, Roggentin P, Mikoleit M, Fields PI, Bockemühl J, Grimont PA, Weill FX. Supplement 2003 - 2007 ( ${ }^{\circ}$ 47) to the WhiteKauffmann -Le Minor scheme. Research in Microbiology. 2010; 161:26-29.

[3] Colla, FL, Rodrigues, LB, Borsoi, A, Dickel, EL, Nascimento, VP, Santos, LR. Isolamento de Salmonella Heidelberg em Diferentes Pontos da 
Tecnologia de Abate de Frangos de Corte. Arquivo Instituto Biológico. 2012; 79 (4):603-606.

[4] Doyle, MP, Erickson, MC. Reducing the carriage of foodborne pathogens in livestock and poultry. Poultry Science. 2006; 85 (6):960-973

[5] Buncic, S, Sofos, J. Interventions to control Salmonella contamination during poultry, cattle and pig slaughter. Food Research International. 2012; 45 (2):641-655.

[6] Mani-López, E, García, HS, López-Malo, A. Organic acids as antimicrobials to control Salmonella in meat and poultry products. Food Research International. 2012; 45 (2): 713-721.

[7] USDA - United States Department of Agriculture. Directive 7120.1, Safe and Suitable Ingredients Used in The Production of Meat, Poultry, and Egg Products, Revision 14, dated March 22, Food Safety and Inspecion Service. FSIS; 2012.

[8] Brasil. Ministério da Agricultura, Pecuária e Abastecimento. Circular no 668 de 19/09/2006. Diretrizes para preparação de Plano de APPCC (HACCP) para o processo de abate de aves. Brasília: 2006. 3 p.

[9] Brasil. Ministério da Agricultura, Pecuária e Abastecimento. Instrução Normativa $\mathrm{N}^{\circ} 70,10$ outubro de 2003. Programa De Redução De Patógenos Monitoramento Microbiológico Controle De Salmonella sp. Em Carcaças De Frangos E Perus. Diário Oficial da República Federativa do Brasil, Poder Executivo, Brasília, DF, 10 out. 2003 a. Seção 1, p. 9.

[10] ISO 6579. Microbiology of food and animal feeding stuffs - Horizontal method of detection of Salmonella spp. 4.ed. 2002. The Internacional Organization for Standardization, Amendment 1.

[11] NCCLS. National Committee for Clinical Laboratory Standards. Performance Standards for Antimicrobial Disk Susceptibility tests; Approved
Standard - Eightth NCCLS. Document M2-A8. Wayne, Pennsylvani, USA, 2003.

[12] Brasil. Ministério da Agricultura e do Abastecimento. Manual de métodos microbiológicos para alimentos. Brasília: Coordenação Geral de Laboratório Animal; 1992.

[13] Silva, N, Junqueira, VCA, Silveira, NFA, Taniwaki, MH, Santos, RFS, Gomes, RAR. Manual de Métodos de Análise Microbiológicas de Alimentos. São Paulo: Varela; 2010.

[14] Harnvajanawong, N, Thongkon, N, Ungchusuk, C, Ramsomphob, W. Effect of Trichloroisocyanuric Acid Disinfectant Filled in Swimming Pool. The Joint International Conference on "Sustainable Energy and Environment (SEE)" 1-3 December; Hua Hin, Thailand. 2004. S005P.

[15] Mattos, A. A. Tratamento de água para abastecimento público com o uso de tabletes de ácido tricloroisocianúrico. IN: Assembléia Nacional da ASSEMAE, ASSEMAE Associação Nacional dos Serviços Municipais de Saneamento. 19 de abril; São Paulo. 2004, p34.

[16] Gonçalves AC, Almeida RCC, Alves, MAO, Almeida PF. Quantitative investigation on the effects of chemical treatments in reducing Listeriamonocytogenes populations on chicken breast meat. Food Control. 2005; 16: 617-622.

[17] Mokgatla, RM, Brozel, VS, Gouws,PA. Isolation of Salmonella resistant to hypochlorous acid from a poultry abattoir. Letters in Applied Microbiology. 1998; 27: 379-382.

[18] Macedo, JAB. O processo de desinfecção pelo uso de derivados clorados em função do $\mathrm{pH}$ e a Portaria 518/2004 do Ministério da Saúde. XLIV Congresso Brasileiro de Química.20 a 24 setembro; Fortaleza-CE. 2004, p1-11.

[19] Tan, SY, Mikš-krajnika, M, Neo, SY, Tan, A, Khoo, GH, Yuk, H-G. Effectiveness of various sanitizer treatments for inactivating natural 
microflora and Salmonella spp. on turnip (Pachyrhizus erosus). Food Control. 2015; 54: 216-224.

[20] Jaenisch, FRF, Kuchiishi, SS, Coldebella, A. Atividade antibacteriana de desinfetantes para uso na produção orgânica de aves. Ciência Rural. 2010; 40 (2):384-388.

[21] Bauermeister LJ, Bowers JW, Townsend JC, Mckee SR. The microbial and quality properties of poultry carcasses treated with peracetic acid as an antimicrobial treatment. Poultry Science. 2008; 87(11):2390-2398.

[22] Machado, TRM, Malheiros, PS, Brandelli, A, Tondo, EC. Avaliação da resistência de Salmonella à ação de desinfetantes ácido peracético, quaternário de amônio e hipoclorito de sódio. Revista do Instituto Adolfo Lutz. 2010; 69 (4): 475-481.

[23] Brul, S.; Coote, P. Preservative agents in foods Mode of action and microbial resistance mechanisms. International Journal of Food Microbiology.1999; 50:1-17.

[24] Burin, RCK, Silva JR, A, Nero, LA. Influence of lactic acid and acetic acid on Salmonella spp. growth and expression of acid tolerance-related genes. Food Research International. 2014; 64: 726-732.

[25] Kang, S, Jang, A, Lee, SO, Min, JS, Kim, S, Lee, M. Effect of organic acids on microbial populations and Salmonella Typhimurium in pork loins. Asian Australasian Journal of Animal Sciences. 2003; 16 (1): 96-99

[26] Steenackers, H, Hermans, K, Vanderleyden, J, DE Keersmaecker, SCJ. Salmonella biofilms: An overview on occurrence, structure, regulation and eradication. Food Research International. 2012; 45 (2): 502-531. 